\title{
Fundamental research for a new confocal line X-ray spectrometer
}

Hitomi Nakano, Shota Sonoda, Tsugufumi Matsuyama, Shintaro Komatani, Kouichi Tsuji

\begin{tabular}{|c|l|}
\hline Citation & X-RAY SPECTROMETRY. 50(3); 224-230 \\
\hline Issue Date & 2021-04-12 \\
\hline Version of Record & 2021-01-05 \\
\hline Type & Journal Article \\
\hline Textversion & author \\
\hline Rights & $\begin{array}{l}\text { This is the peer reviewed version of the following article: X-RAY } \\
\text { SPECTROMETRY. Vol.50, Issu.3, 224-230, which has been published in final } \\
\text { form at https://doi.org/10.1002/xrs.3218. This article may be used for } \\
\text { non-commercial purposes in accordance with Wiley Terms and Conditions for } \\
\text { Use of Self-Archived Versions. This article may not be enhanced, enriched or } \\
\text { otherwise transformed into a derivative work, without express permission from } \\
\text { Wiley or by statutory rights under applicable legislation. Copyright notices } \\
\text { must not be removed, obscured or modified. The article must be linked to } \\
\text { Wiley's version of record on Wiley Online Library and any embedding, framing } \\
\text { or otherwise making available the article or pages thereof by third parties from } \\
\text { platforms, services and websites other than Wiley Online Library must be } \\
\text { prohibited. }\end{array}$ \\
\hline DOI & \begin{tabular}{l} 
10.1002/xrs.3218 \\
\hline
\end{tabular} \\
\hline
\end{tabular}

\section{Self-Archiving by Author(s)}

Placed on: Osaka City University Repository 


\title{
Fundamental research for a new confocal line X-ray spectrometer
}

\author{
Hitomi Nakano ${ }^{1,2}$, Shota Sonoda ${ }^{2}$, Tsugufumi Matsuyama ${ }^{2}$, Shintaro Komatani ${ }^{1}$, Kouichi \\ Tsuji $^{2}$ \\ ${ }^{1}$ HORIBA TECHNO SERVICE Co., Ltd Analytical Technology Center, \\ 2 Kisshoin-Miyanohigashi-cho, Minami-ku, Kyoto, Kyoto, 601-8305 Japan \\ ${ }^{2}$ Department of Applied Chemistry \& Bioengineering, Graduate School of Engineering, Osaka City \\ University, 3-3-138 Sugimoto-cho, Sumiyoshi-ku, Osaka, Osaka 558-8585, Japan
}

\begin{abstract}
Confocal X-ray fluorescence spectrometry can be used to perform three-dimensional elemental analysis, which is impossible using general micro X-ray fluorescence (XRF) spectrometry without any collimating optics in the detection channel. In this study, we designed, for the first time, a new confocal line XRF (C-L-XRF) system that can obtain elemental information of a larger area and higher intensity in XRF analysis than conventional confocal point XRF (C-P-XRF) analysis. We evaluated the basic performance of C-L-XRF, such as XRF intensity and horizontal and depth spatial resolutions. We identified that the spatial resolution of C-L-XRF in the horizontal and depth resolutions is approximately 2.9 and 2.6 times, respectively, compared to those of conventional C-P-XRF. However, it is possible to obtain an XRF intensity that is approximately 33 times higher than C-P-XRF intensity. C-L-XRF is expected to be effective for analyzing wide-area samples such as layered samples.
\end{abstract}

\section{KEYWORDS}

Confocal line XRF, Micro XRF, Non-destructive analysis, Depth analysis

\section{INTRODUCTION}

X-ray fluorescence (XRF) spectrometry enables nondestructive elemental analysis ${ }^{[1-3]}$. In general XRF microscopy, primary X-rays irradiate a sample, and subsequently, a detector above the sample detects the fluorescence X-rays secondarily generated from the elements of the sample. Because fluorescence X-rays from the inner area are absorbed by coexisting elements, we cannot perform depth-selective measurements. Meanwhile, in confocal point X-ray fluorescence (C-P-XRF) analysis, polycapillary lenses are placed in front of the X-ray generator and detector ${ }^{[4]}$ to detect X-rays from only the confocal volume ${ }^{[5]}$. Depth-selective analysis and three-dimensional analysis can be performed ${ }^{[6]}$ 
by scanning the sample stage.

Several studies regarding C-P-XRF have been performed and reported.

The concept of confocal XRF was proposed in 1990 by the assembly of capillaries ${ }^{[4]}$. Subsequently, this technique, proposed by Kumakhov, has been applied to synchrotron radiations ${ }^{[6]}$ and desktop XRF devices ${ }^{[5]}$, as it enabled the measurement of depth elemental profiles in various samples ${ }^{[7-12]}$. Basic research on quantitative analysis with confocal XRF has also been conducted, and various studies are underway ${ }^{[11-14]}$.

However, the X-ray intensity from the deeper region will be lower owing to their absorption by the matrix elements. Therefore, a significant amount of time is required to obtain sufficient XRF intensities from the C-P-XRF.

In this study, a novel measurement technique for a layered sample was developed using a new optical system that utilizes thin sheet-type X-ray beams. The sheet-type X-ray beams were formed by placing Soller slits on the front of the X-ray tube and detector. This system, which extracts elemental information in the overlapped area of both the Xray beam and detector, was named as the confocal line XRF (C-L-XRF) micro XRF system. Thus, we believe that this technique will be useful for obtaining the average elemental information of a determined depth in the layered sample.

In this study, we evaluated the basic performance of C-L-XRF in comparison with C-PXRF. In C-P-XRF, a monochromatic micro X-ray source, equipped with a synthetic multilayer assembly in a side-by-side Kirkpatrick-Baez (K-B) mirror, was used as the Xray generator ${ }^{[15,16]}$.

\section{EXPERIMENTAL ANALYSIS}

\section{2-1. Design of the C-P-XRF}

Experiments were performed using prototyped instruments at the Osaka City University [15]. A schematic of the C-P-XRF system is shown in Fig. 1. This optical system was developed based on the previous C-P-XRF ${ }^{[17,18]}$. It comprises an X-ray generator, a polycapillary lens, sample stage, and detector. We used a monochromatic micro X-ray source equipped with a Mo X-ray tube target (Rigaku Corporation, Japan) and a synthetic multilayer assembly in a side-by-side K-B mirror ${ }^{[16]}$. A polycapillary lens was set in front of a silicon drift detector (SDD, detector area =50 mm², energy resolution=150 eV@Mn $\mathrm{K} \alpha$, thickness $=0.5 \mathrm{~mm}$, Techno X. Ltd). The nominal focal distance and nominal beam size at the focus of the K-B mirror were $150 \mathrm{~mm}$ and $41 \mu \mathrm{m}$ respectively ${ }^{[15]}$.

\section{2-2. Design of the C-L-XRF}

A schematic of the C-L-XRF system is shown in Fig. 2. The system comprises an X-ray 
generator, Soller slits ${ }^{[19]}$ for aligning the incident X-rays and generating X-rays in , a sample stage capable of moving in the $\mathrm{X}$ and $\mathrm{Y}$ directions, and a detector. An enclosed Xray tube for X-ray diffraction (Rigaku: target Mo) was used as the X-ray generator. The size of the X-ray can be determined by placing slits in front of the generator and detector. In this study, the slit in front of the X-ray tube was $100 \mu \mathrm{m} \times 12 \mathrm{~mm}$, while the slit on the detector side was $50 \mu \mathrm{m} \times 12 \mathrm{~mm}$. The Soller slit for collimating the X-rays was $100 \mu \mathrm{m}$ wide. The detector used was an SDD Vortex EX-60 (element area $=50 \mathrm{~mm}^{2}$, thickness $=0.5 \mathrm{~mm}$, energy resolution $\mathrm{Mn} \mathrm{K \alpha}=130 \mathrm{eV}$, Hitachi High-Technologies Corporation, Japan). Because the sample stage can be moved along the $\mathrm{X}$ and $\mathrm{Y}$ axes, the depth of focus can be selected.

In this study, the primary X-rays were shaped linearly by passing them through the Soller slits and other slits. Furthermore, the fluorescent X-rays emitted from the sample were detected linearly using the same method, thereby enabling a "confocal line" analysis. Because the X-rays formed a linear shape instead of a point, their intensity was expected to increase compared to the C-P-XRF analysis, accompanied by a reduction in the measurement time. However owing to the larger spatial resolution in the horizontal direction (beam size) compared to C-P-XRF, it is considered unsuitable for the measurement of minute particles and more effective for the analysis of large samples such as layered samples. The other advantages of the C-L-XRF designed in this study are its lower assembly cost and the larger working distance of the Soller slit, which facilitates the analysis of a large area.

\section{2-3. Evaluation of spatial resolution (horizontal and depth)}

A schematic representation of the evaluation of the horizontal spatial resolution is shown in Fig. 3. To evaluate the horizontal spatial resolution, a W wire with a thickness of $5 \mu \mathrm{m}$ for the C-P-XRF system and a Ni wire with a thickness of $25 \mu \mathrm{m}$ for the C-L-XRF system were used. The wires were slid horizontally across while focusing the X-rays, and the changes in the W L $\alpha$ XRF intensity in the C-P-XRF and Ni K $\alpha$ intensity in the C-L-XRF were measured. The net intensities were obtained by Gaussian fitting of the element peaks and separating the background and peak intensities. From the full width at half maximum (FWHM) of the X-ray intensity profile obtained, the X-ray beam sizes were calculated according to equation (1). Here, $S_{b}$ represents the horizontal spatial resolution (X-ray beam size), $S$ represents the FWHM divided by the square root of 2 considering the Xray incident angle $\left(45^{\circ}\right)$, and $T_{\text {wire }}$ represents the wire diameter ${ }^{[3]}$. As the X-rays were incident at an angle of $45^{\circ}$, the obtained FWHM value was divided by the square root of 2 before substituting into equation (1). The measurement conditions in the C-P-XRF 
system were as follows: $\mathrm{X}$-ray tube voltage $=50 \mathrm{kV}$, tube current $=0.4 \mathrm{~mA}$, and measurement interval $=2 \mu \mathrm{m}$. Furthermore, measurements were performed for $30 \mathrm{~s}$ per point. The C-L-XRF measurement conditions were as follows: X-ray tube voltage $=20$ $\mathrm{kV}$, tube current $=20 \mathrm{~mA}$, and measurement interval $=4 \mu \mathrm{m}$. These measurements were performed for $10 \mathrm{~s}$ per point.

$$
S_{b}=\sqrt{S^{2}-T_{\text {wire }}^{2}}
$$

The depth spatial resolution was evaluated as follows. Fig. 4 shows a schematic for evaluating the measurement of the depth spatial resolution. The evaluation was performed using the same calculation for the beam size, employing a sample with a layered structure, in which a metal was vapor-deposited at a thickness of $500 \mathrm{~nm}$ from the surface of a $\mathrm{Si}$ wafer ${ }^{[14]}$. The sample was set on a sample table, and the stage was moved to the depth direction. The depth spatial resolution was obtained by performing measurements while scanning the focus and obtaining the FWHM of the X-ray intensity of the layer element. Depth spatial resolution was calculated using equation (2), where $S_{d}$ represents the depth spatial resolution, $S$ represents the FWHM, and $T_{\text {layer }}$ represents the layer thickness ${ }^{[3,14]}$. Because the thickness of the metal layer is considerably lower than the value of FWHM, it does not need to be considered in the calculation.

$$
S_{d}=\sqrt{S^{2}-T_{\text {layer }}^{2}}
$$

The C-P-XRF system measures thin layers of the metals $\mathrm{Ti}, \mathrm{Fe}, \mathrm{Ni}, \mathrm{Cu}$, and $\mathrm{Au}$ under the following conditions: X-ray tube voltage $=50 \mathrm{kV}$, tube current $=0.4 \mathrm{~mA}$, measurement time $=15 \mathrm{~s}$, and measurement interval $=1 \mu \mathrm{m}$. Meanwhile, the C-L-XRF system measures thin layers of the metals $\mathrm{Cr}, \mathrm{Ni}, \mathrm{Cu}$, and $\mathrm{Au}$ under the following conditions: voltage $=40 \mathrm{kV}$, current $=20 \mathrm{~mA}$, measurement time $=15 \mathrm{~s}$, and measurement interval $=2 \mu \mathrm{m}$.

\section{2-4. Measurement of a layered sample}

We measured a leather sample (Cordovan) using both C-P-XRF and C-L-XRF. The sample, which had a layered structure, was provided by the Department of Research of the Hyogo Prefectural Police. The dyed side of the leather, which was regarded as the sample surface, was rich in $\mathrm{Zn}$ whereas the bulk of the sample was rich in Fe. A crosssectional image of the sample is shown in Fig. 5. The C-P-XRF measurement was performed while scanning the sample in the depth direction under the following conditions: X-ray tube voltage $=50 \mathrm{kV}$, current $=0.4 \mathrm{~mA}$, measurement area $=510 \mu \mathrm{m}$, interval distance $=30 \mu \mathrm{m}$, and measurement time $=2500 \mathrm{~s}$. The experimental conditions 
for the measurement for C-L-XRF were as follows: X-ray tube voltage $=40 \mathrm{kV}$, current $=20 \mathrm{~mA}$, measurement area $=420 \mu \mathrm{m}$, interval distance $=60 \mu \mathrm{m}$, and measurement time $=150 \mathrm{~s}$.

\section{RESULTS AND DISCUSSION}

\section{3-1. Evaluation of spatial resolution (horizontal and depth)}

The results of the evaluation of the horizontal spatial resolutions are shown below. Fig. 6 shows the X-ray intensity profiles for the W L $\alpha$ plot of the C-P-XRF when the W wire sample was scanned horizontally and the Ni K $\alpha$ plot of the C-L-XRF when the Ni wire sample was scanned horizontally. The FWHM of the C-P-XRF was calculated as 53.4 $\mu \mathrm{m}$. Considering the detection angle $\left(45^{\circ}\right.$ ), the value of $S$ obtained by dividing the FWHM value by the square root of 2 was $37.8 \mu \mathrm{m}$. The beam size $\left(S_{b}\right)$ was estimated to be $37.5 \mu \mathrm{m}$ using equation (1), with the appropriate values for the wire thickness $\left(T_{\text {wire }}\right)$, detection angle $\left(45^{\circ}\right)$, and FWHM of the W L $\alpha$ intensity profile. Meanwhile, the FWHM of C-L-XRF was calculated to be $155 \mu \mathrm{m}$. Considering the detection angle $\left(45^{\circ}\right)$, the value of $S$ obtained by dividing the FWHM value by the square root of 2 was $110 \mu \mathrm{m}$. The beam size $\left(S_{b}\right)$ was calculated to be $107 \mu \mathrm{m}$, using equation (1), with the appropriate values for the wire thickness ( $\left.T_{\text {wire }}\right)$, detection angle $\left(45^{\circ}\right)$, and FWHM of the Ni K $\alpha$ intensity profile. Considering the width of the slit installed in front of the $\mathrm{X}$-ray generator and detection angle, the value obtained using C-L-XRF is considered to be appropriate.

The results of the evaluation of the depth spatial resolutions are shown below. The XRF intensity profiles of the $\mathrm{Au} \mathrm{L} \alpha$ intensities for C-P-XRF and C-L-XRF are shown in Fig. 7. The depth spatial resolutions were estimated as $24 \mu \mathrm{m}$ and $62 \mu \mathrm{m}$ at $\mathrm{Au} \mathrm{L} \alpha$ for C-P$\mathrm{XRF}$ and C-L-XRF, respectively, using equation (2). Considering the width of the slit installed in front of the X-ray detector and detection angle, the value obtained using C-L$\mathrm{XRF}$ is considered to be better than the expected value.

The graph of the relationships between the energy of XRF of each element and the depth resolutions of C-P-XRF and C-L-XRF is shown in Fig. 8. In C-P-XRF, the depth resolution values were smaller when the fluorescence $\mathrm{X}$-ray energy was higher. Because polycapillary lenses were used for X-ray focusing in confocal XRF, the total reflection critical angle on the inner wall of the capillary has energy dependence. Therefore, the Xrays on the low-energy side have a larger beam diameter spread, and the X-rays on the high-energy side have a smaller beam diameter. By contrast, in the C-L-XRF system, the spatial resolution had no energy dependence. This is because total internal reflection did 
not occur on the inner wall of the Soller slit, and only X-rays passed between the slits.

\section{3-2. Measurement of a layered sample}

The results of measurements of the XRF intensity of the profiles of $\mathrm{Zn}$ and $\mathrm{Fe}$ in the leather samples obtained by C-P-XRF and C-L-XRF are shown in Fig. 9. It was confirmed that the intensity of $\mathrm{Zn}$ was higher at the surface of the leather, whereas the intensity of $\mathrm{Fe}$ intensity was higher in deeper area of the sample. In C-L-XRF, the intensity of Fe began to increase before the intensity of $\mathrm{Zn}$ was reduced significantly. According to the graph, upon comparing the $\mathrm{Zn}$ intensities at the depth where the highest intensities were obtained, C-L-XRF showed an intensity of approximately $0.67 \mathrm{cps} / \mathrm{mA}$, whereas that for $\mathrm{C}-\mathrm{P}-\mathrm{XRF}$ was approximately $0.02 \mathrm{cps} / \mathrm{mA}$. The intensity of C-L-XRF was confirmed to be approximately 33 times higher than that of C-P-XRF.

Since the spatial resolution depends on the slit size, the spatial resolution of C-L-XRF is lower than that of C-P-XRF, but better spatial resolution can be obtained by using thinner slits.

\section{CONCLUSION}

In this study, we designed a new C-L-XRF system and evaluated its basic performance. Primary X-rays were shaped linearly by passing them through Soller slits and other slits, and the fluorescent X-rays emitted from the sample were detected linearly by the same method. This enabled the "confocal line" analysis. We confirmed that the horizontal resolution of the C-L-XRF was $107 \mu \mathrm{m}$, which was approximately 2.9 times that of the $\mathrm{C}-\mathrm{P}-\mathrm{XRF}$, and the depth resolution of C-L-XRF was approximately $62 \mu \mathrm{m}$, which was nearly 2.6 times that of the C-P-XRF. Furthermore, we confirmed that the spatial resolution of C-L-XRF has no energy dependence. Although, the XRF intensity of C-LXRF was approximately 33 times that of C-P-XRF. Therefore, C-L-XRF appears unsuitable for the elemental analysis of minute areas when compared with C-P-XRF. However, it appears to be effective for wide-area sample measurements such as layered samples.

One of the goals of this study is the quantitative analysis of C-L-XRF. When performing quantification using confocal XRF, it is necessary to correct the effects of self-absorption and secondary excitation inside the sample. Unfortunately, such detailed quantification by confocal XRF has not been completed. Malzer compared confocal XRF with conventional XRF, and Tsuji suggests that it could be useful to treat the sample as an aggregate of layers (mosaic model) and discuss the effects of secondary excitation.

This paper presents a preliminary study on C-L-XRF analysis; however, considering 
how the effects of self-absorption and secondary excitation in the confocal XRF remain to be discussed, we would consider focusing on the development of the quantitative analysis in future work.

\section{REFERENCES}

[1] K.H.A. Janssens, F.C.V. Adams, A. Rindby (Eds.), Microscopic X-ray Fluorescence Analysis, John Wiley \& Sons Ltd, Chichester, 2000.

[2] B. Beckhoff, B. Kanngießer, N. Langhoff, R. Wedell, H. Wolff (Eds), Handbook of Practical X-ray Fluorescence Analysis, Springer, Berlin Heidelberg, 2006.

[3] K. Tsuji, J. Injuk, R. V. Grieken (Eds), X-Ray Spectrometry: Recent Technological Advances, Wiley, John Wiley \& Sons Ltd, Chichester, 2004.

[4] M.A. Kumakhov, F. F. Komarov, "Multiple reflection from surface X-ray optics" Physics Reports (Review Section of Physics Letters), 1990, 191, 289-350.

[5] X. Ding, N. Gao, G. Havrilla, "Monolithic polycapillary x-ray optics engineered to meet a wide range of applications," Proceedings of SPIE, 2000, 4144, 174.

[6] B. Kanngießer,W. Malzer, I. Reiche, A new 3D micro X-ray fluorescence analysis setup - first archaeometric applications, Nucl. Instrum. Methods Phys. Res., 2003, Sect. B 211, 259-264.

[7] Z. Smit, K Janssens, K. Proost, I. Langus, "Confocal $\mu$-XRF depth analysis of paint layers," Nucl. Instr. and Meth. in Phys. Res. 2004, B 219-220, 35-40.

[8] B. Kanngieber, W. Malzer, A. F. Rodriguez, I. Reiche, “Three-dimensional microXRF investigations of paint layers with a tabletop setup," Spectrochimica Acta, 2005, Part B 60. 41-47.

[9] L. Vincze, B. Vekemans, F.E. Brenker, G. Falkenberg, K. Rickers, A. Somogyi, M. Kersten, F. Adams "Three-dimensional trace element analysis by confocal X-ray microfluorescence imaging", Anal. Chem, 2004, 76, 6786-6791.

[10] B. M. Patterson, J. Campbell, G. J. Havrilla, "Integrating 3D images using laboratory-based micro X-ray computed tomography and confocal X-ray fluorescence techniques,” X-ray Spectrom, 2010, 39, 184-190.

[11] W. Malzer, B. Kanngieber, "A model for the confocal volume of 3D micro X-ray fluorescence spectrometer," Spectrochimica Acta, 2005, Part B 60, 1334-1341.

[12] I. Mantouvalou, T. Lachmann, S. P. Signh, K. Vogel-Mikus, B. Kanngieber, "Advanced Absorption Correction for 3D Elemental Images Applied to the Analysis of Pearl Millet Seeds Obtained with a Laboratory Confocal Micro X-ray Fluorescence Spectrometer," Anal. Chem, 2017, 89, 5453-5460.

[13] K. Tsuji, A. Tabe, P. Wobrauscheck, C. Streli, "Secondary excitation process for 
quantitative confocal 3D-XRF analysis," Powder Diffraction, 2015, 30, 2, 109-112. [14] N. Kawahara, T. Matsuno, Y. Takimoto, K. Tsuji, "Secondary excitation observed in lateral scan using confocal micro-XRF,“ Advances in X-ray Analysis, 2016, 59, 7784.

[15] N. Kawahara, S. Sonoda, S. Mita, T. Matsuyama, and K. Tsuji, "Monochromatic Confocal Micro X-ray Fluorescence Spectrometry,” Advances in X-ray Analysis, 2020, $63,125-131$.

[16] P. Kirkpatrick, and A. V. Baez, "Formation of Optical Images by X-rays", J. Opt. Soc. Am, 1948, 38, 766-774.

[17] K. Nakano and K. Tsuji, J. Anal. At. Spectrom, 2010, 25, 562.

[18] T. Nakazawa, and K. Tsuji, "Depth-selective elemental imaging of microSD card by confocal micro-XRF analysis," X-Ray Spectrom, 2013, 42, 123-127.

[19]W. Soller, “A new Precision X-ray Spectrometer,” Phys.Rev, 1924, 24, 158.

\section{Figure captions}

Fig. 1 Schematic of the C-P-XRF system.

Fig. 2 (a) Schematic of the top view of the C-L-XRF system, (b) analysis area in the CL-XRF arrangement. The dotted ellipse shows the confocal line area.

Fig. 3 Schematic of the measurement of the beam size by (a) C-P-XRF and (b) C-LXRF.

Fig. 4 Experimental methods for measuring depth resolution using (a) C-P-XRF and (b) C-L-XRF. The sample was scanned in the depth direction from the X-ray tube and detector. After the depth profiles were obtained, Gaussian fitting was performed to obtain the FWHM.

Fig. 5 Photographs of the leather sample: (a) top and (b) cross-sectional views of the surface.

Fig. 6 XRF intensities of (a) W L $\alpha$ profile of C-P-XRF and (b) Ni K $\alpha$ profile of C-L$\mathrm{XRF}$ obtained for wires which thickness of $5 \mu \mathrm{m}$ and $25 \mu \mathrm{m}$, respectively. 
Fig. 7 XRF intensity of Au profiles of (a) C-P-XRF, and (b) C-L-XRF obtained for Au layer which thickness of $500 \mathrm{~nm}$.

Fig. 8 Relationship between the X-ray energy and depth resolution using (a) C-P-XRF and (b) C-L-XRF.

Fig. 9 Intensity profiles of the Fe $\mathrm{K} \alpha$ and $\mathrm{Zn} \mathrm{K} \alpha$ intensities obtained using (a) C-P-XRF and (b) C-L-XRF for the leather sample (Cordovan). Red symbols shows the $\mathrm{Zn} \mathrm{K \alpha}$ and black symbol shows the Fe Ka intensities. 


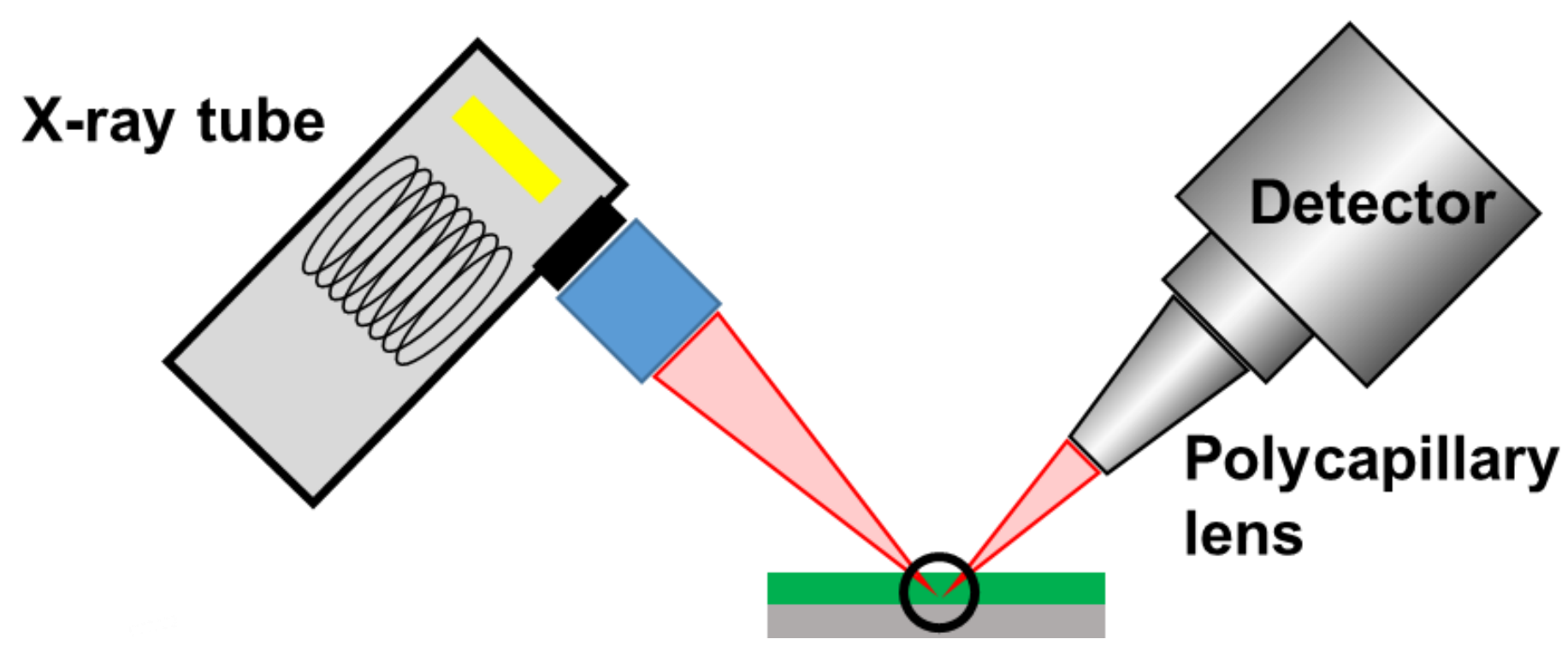

Fig. 1 Schematic of the C-P-XRF system. 
(a)

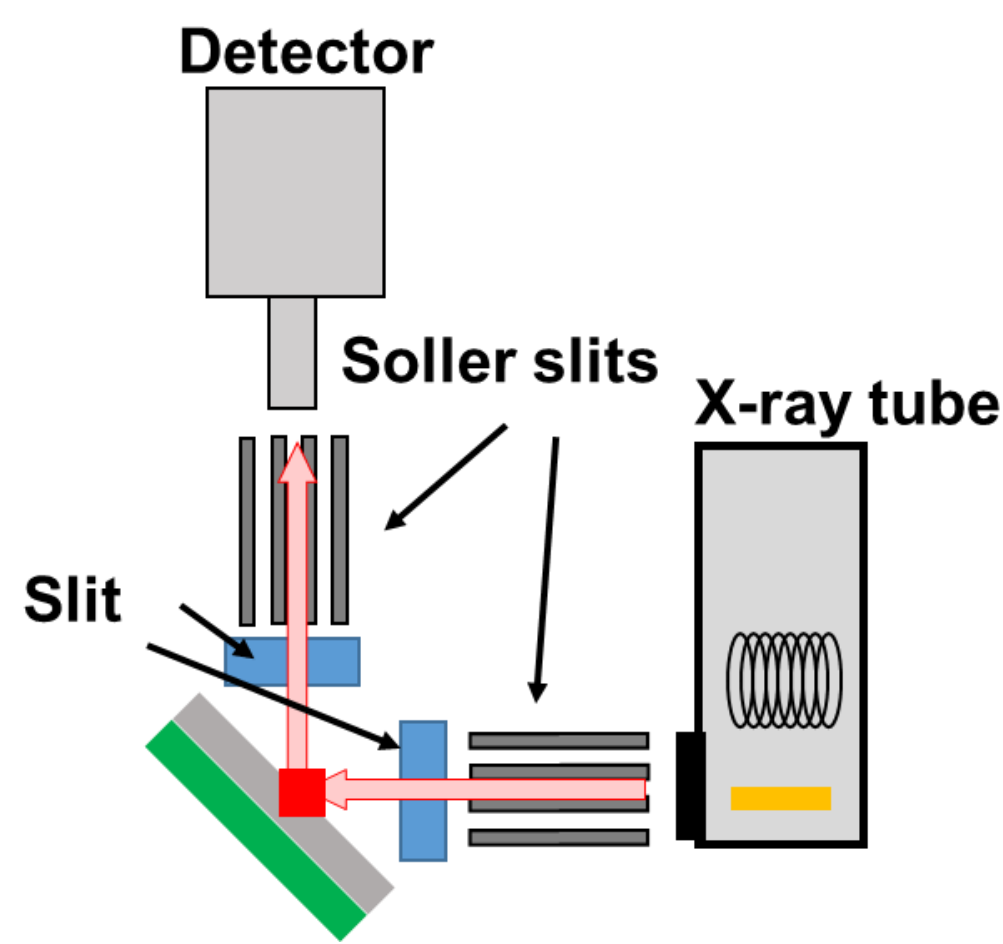

(b)

\section{Primary X-rays X-ray fluorescence Soller slit}

Soller slit

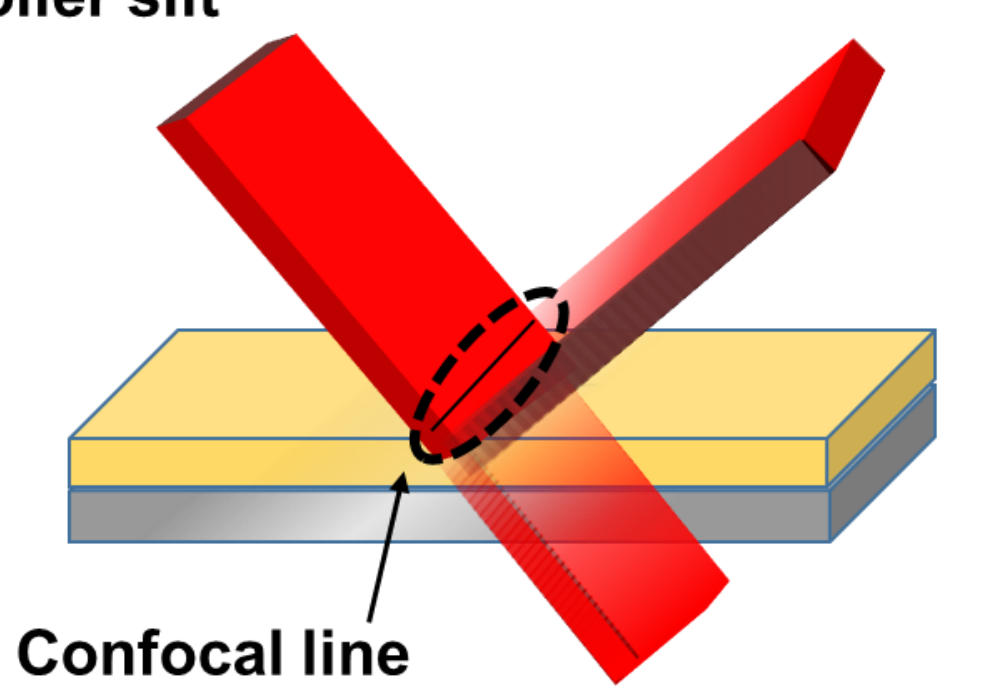

Fig. 2 (a) Schematic of the top view of the C-L-XRF system, (b) analysis area in the C-L-XRF arrangement. The dotted ellipse shows the confocal line area. 
(a)

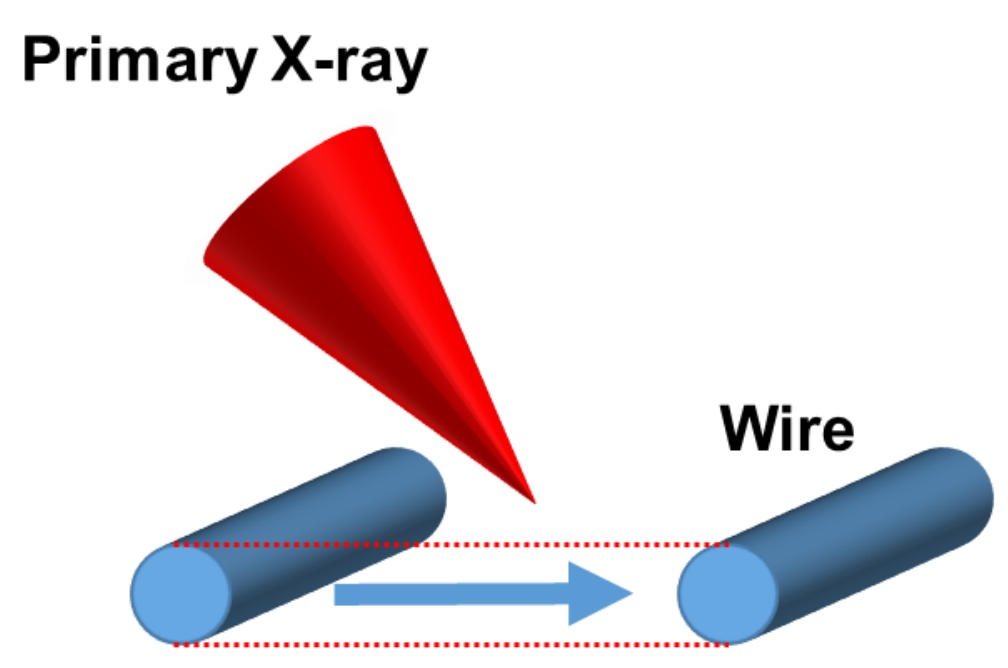

(b)

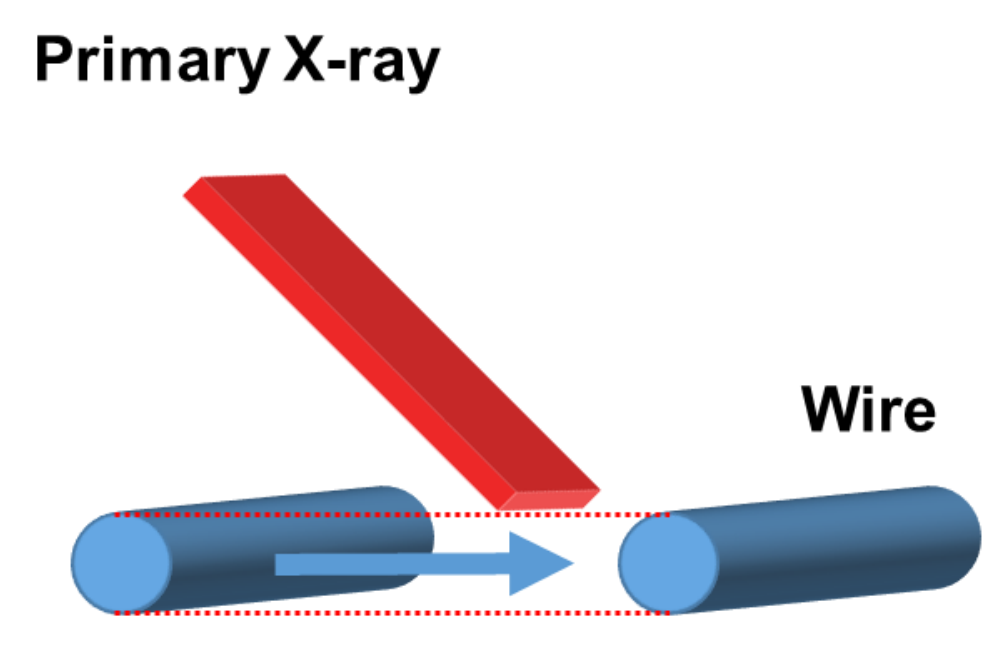

Fig. 3 Schematic of the measurement of the beam size by (a) C-P-XRF and (b) C-L-XRF. 
(a)

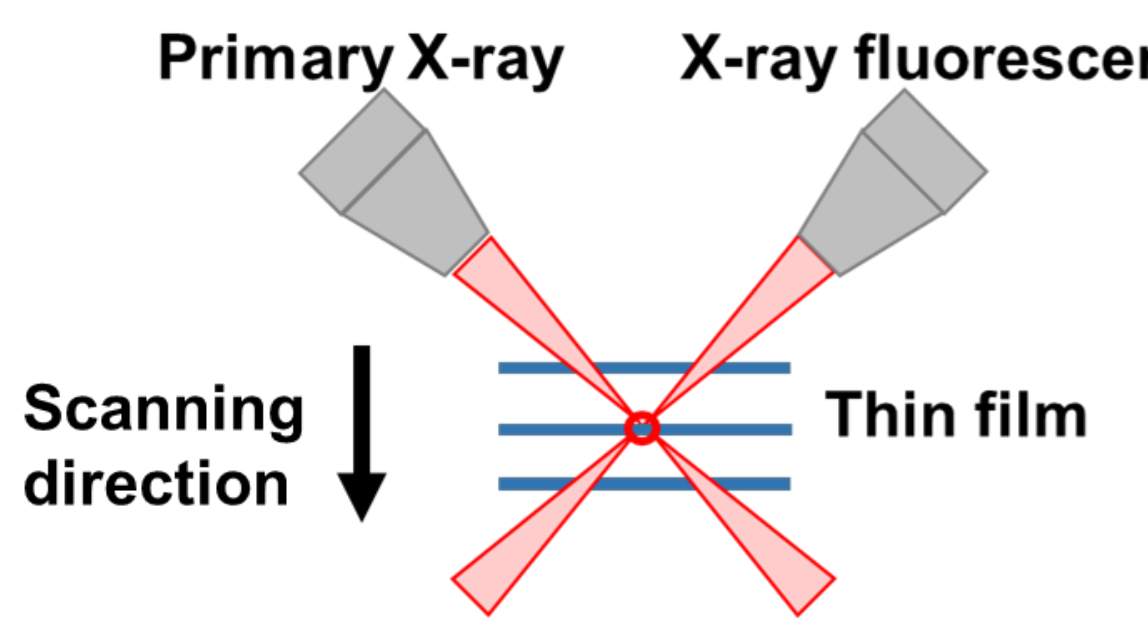

(b)

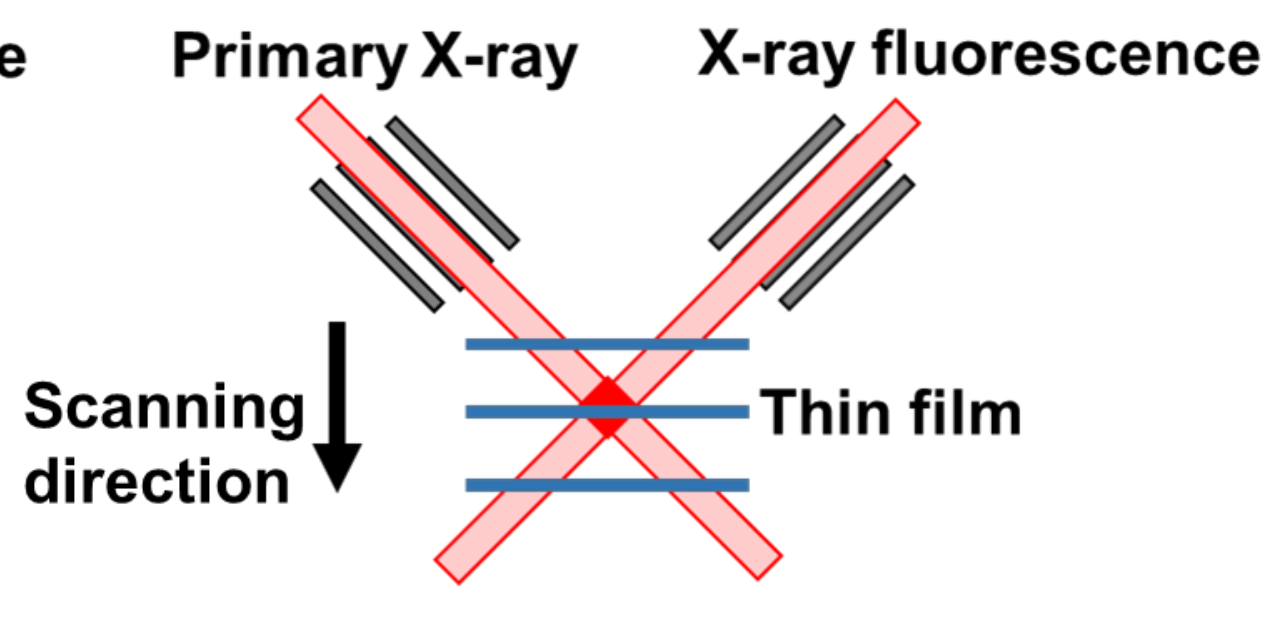

Fig. 4 Experimental methods for measuring depth resolution using (a) C-P-XRF and (b) C-L-XRF. The sample was scanned in the depth direction from the X-ray tube and detector. After the depth profiles were obtained, Gaussian fitting was performed to obtain the FWHM. 
(a)

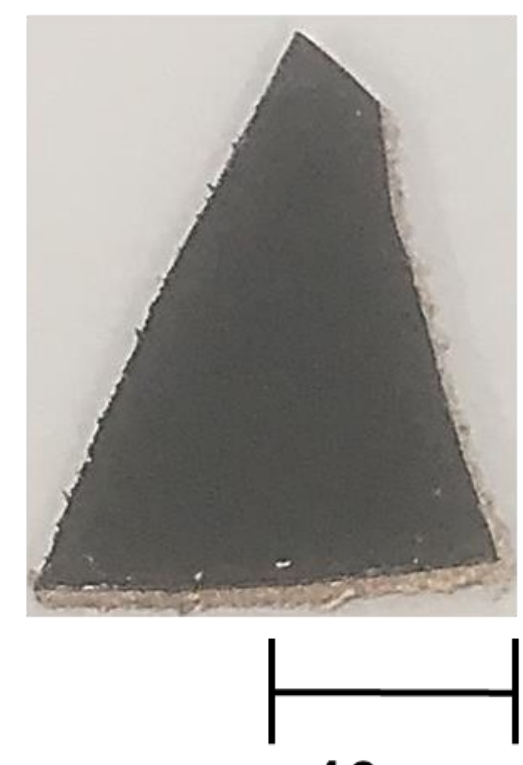

$10 \mathrm{~mm}$ (b)

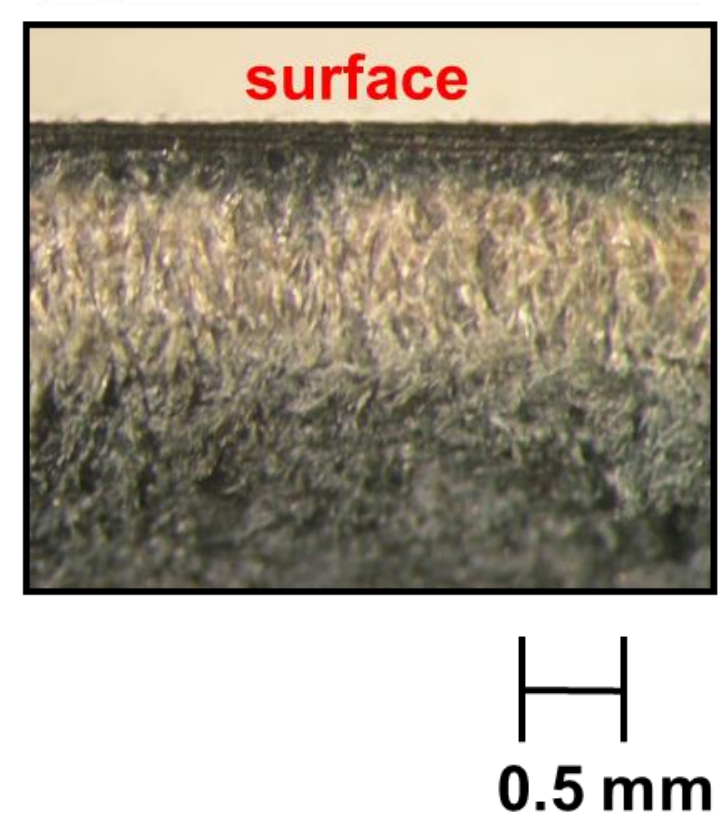

Fig. 5 Photographs of the leather sample: (a) top and (b) cross-sectional views of the surface. 
(a) Net intensity (cps)

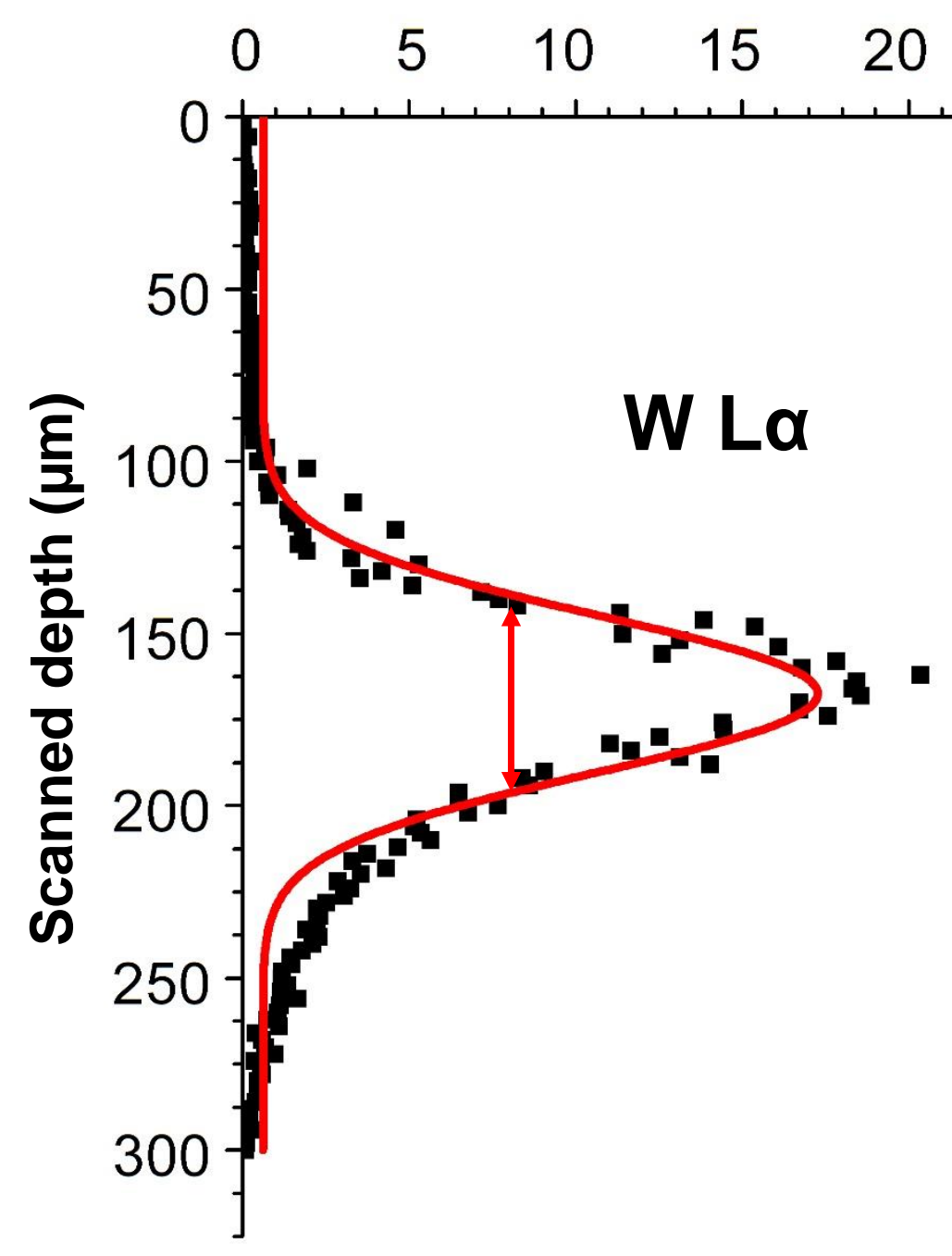

(b) Net intensity (cps)

25

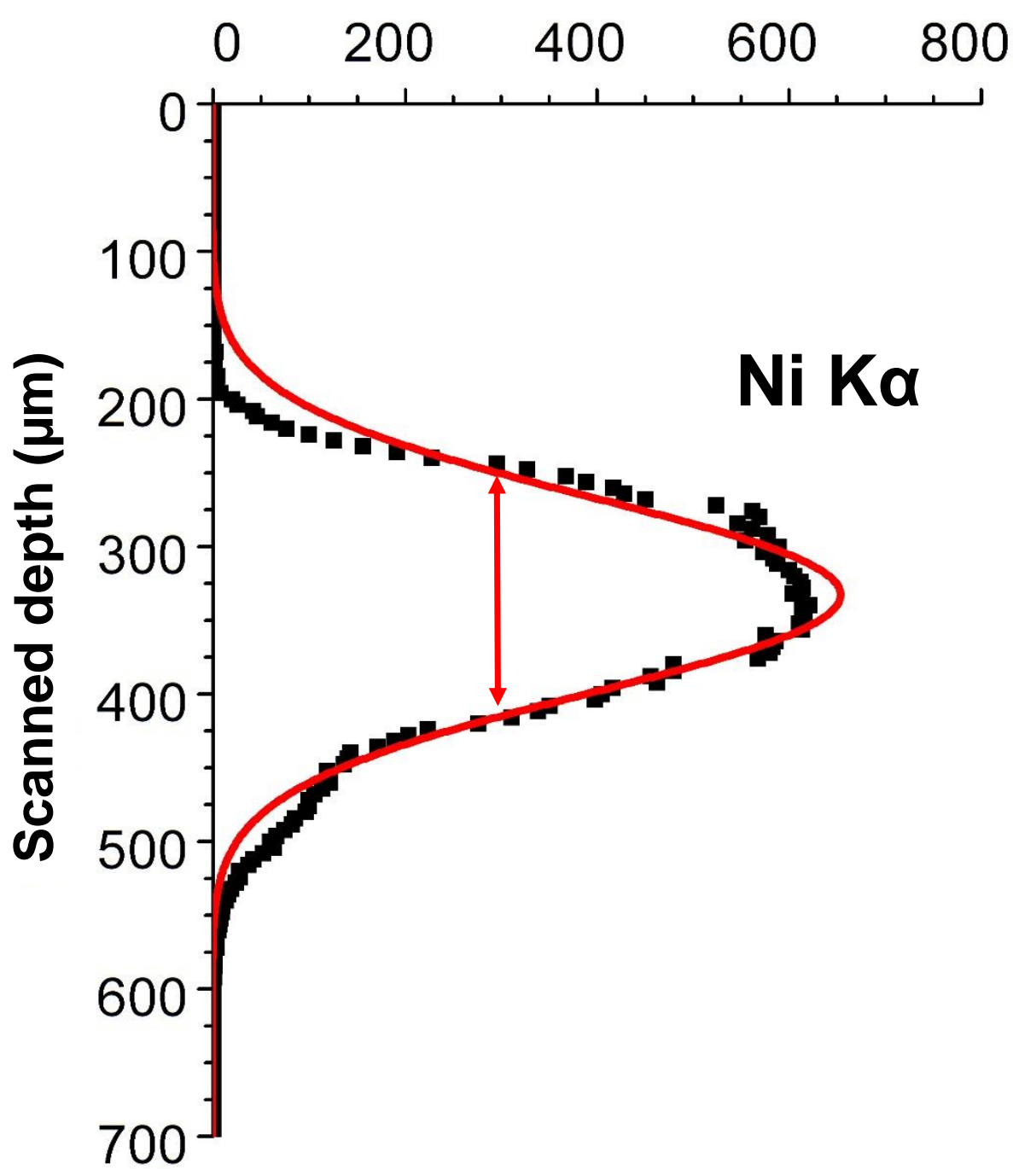

Fig. 6 XRF intensities of (a) W L $\alpha$ profile of C-P-XRF and (b) Ni K $\alpha$ profile of C-L-XRF obtained for wires which thickness of $5 \mu \mathrm{m}$ and $25 \mu \mathrm{m}$, respectively. 
(a)

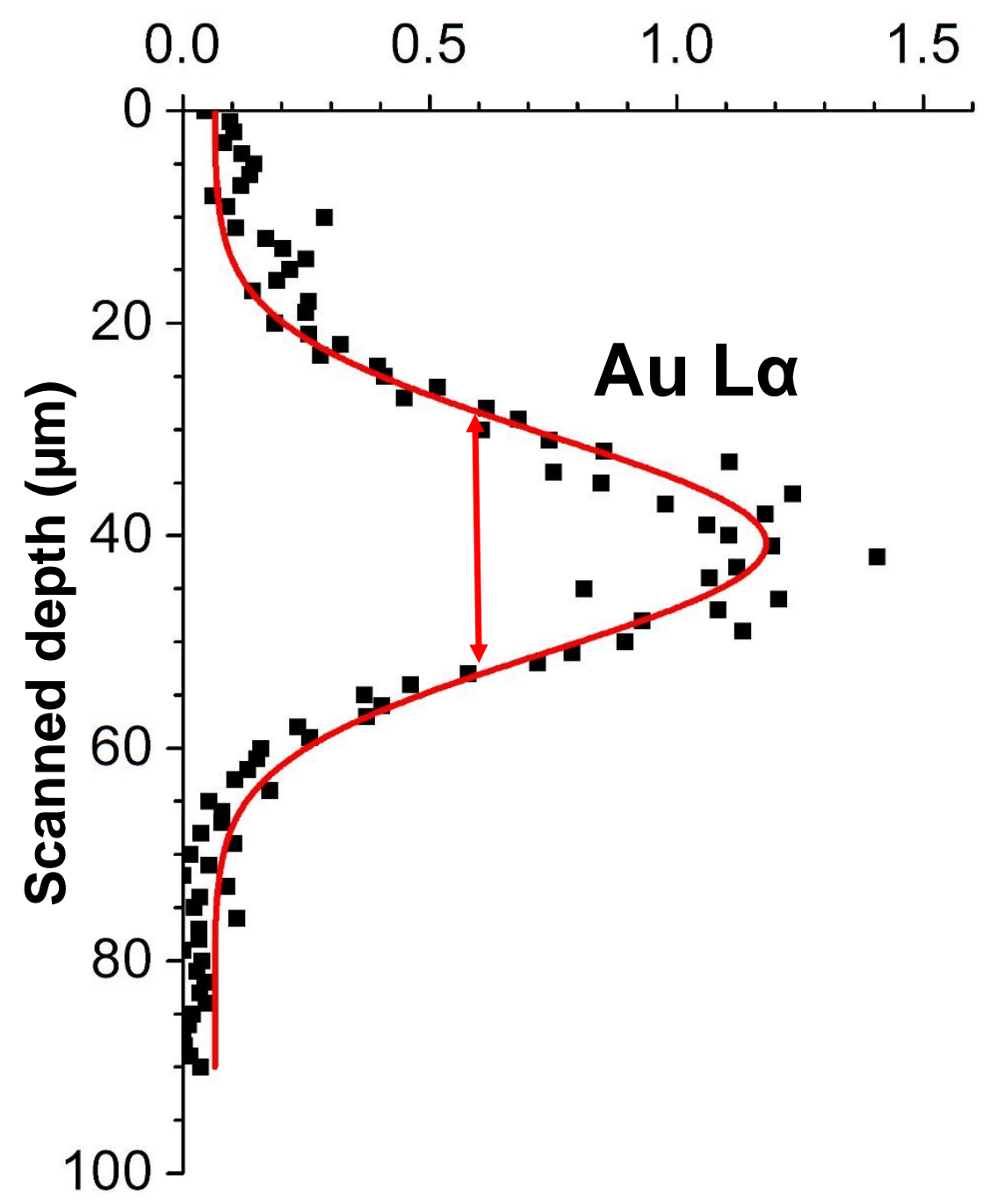

(b) Net intensity (cps)

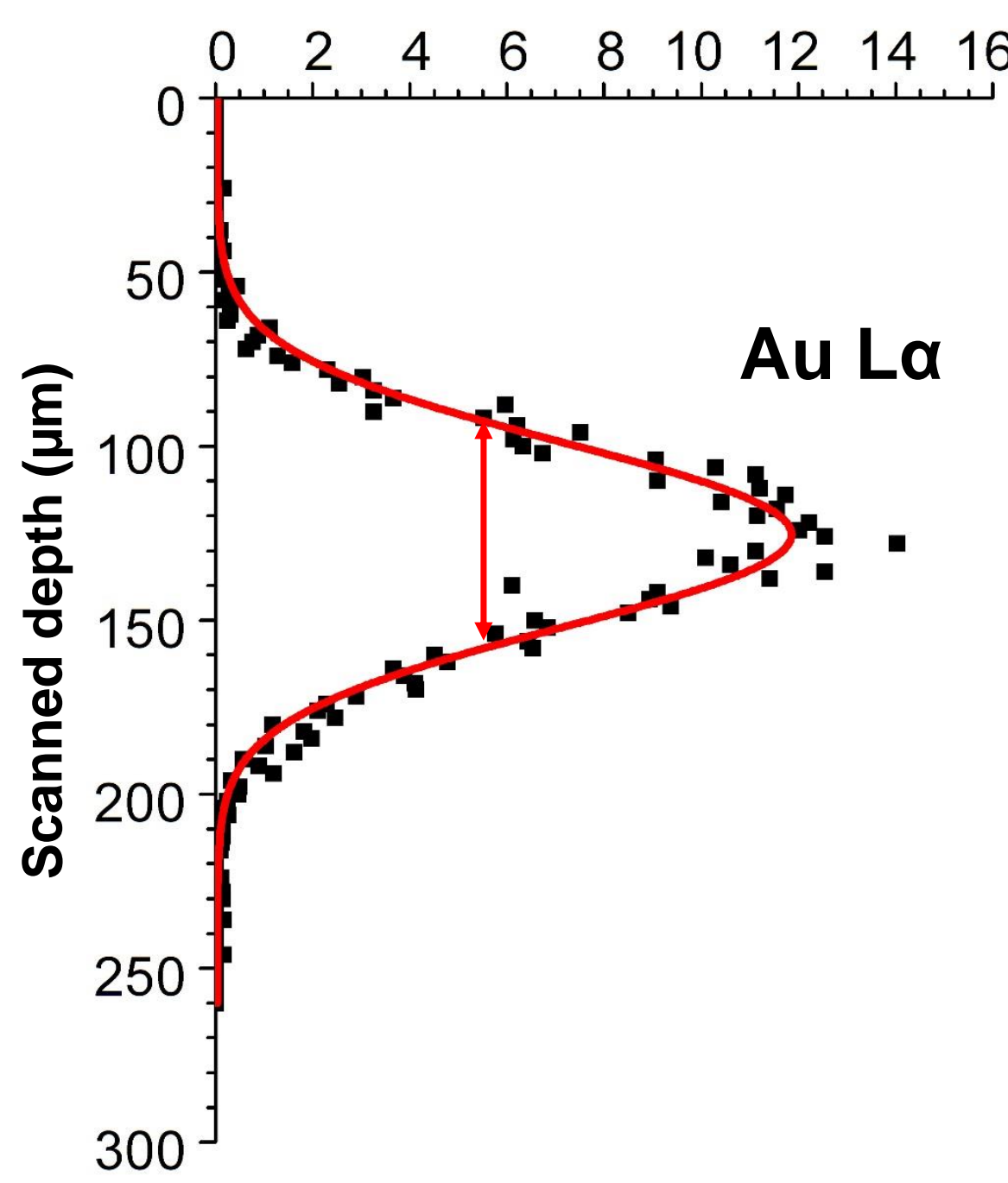

Fig. 7 XRF intensity of Au profiles of (a) C-P-XRF, and (b) C-L-XRF obtained for Au layer which thickness of $500 \mathrm{~nm}$. 
(a)

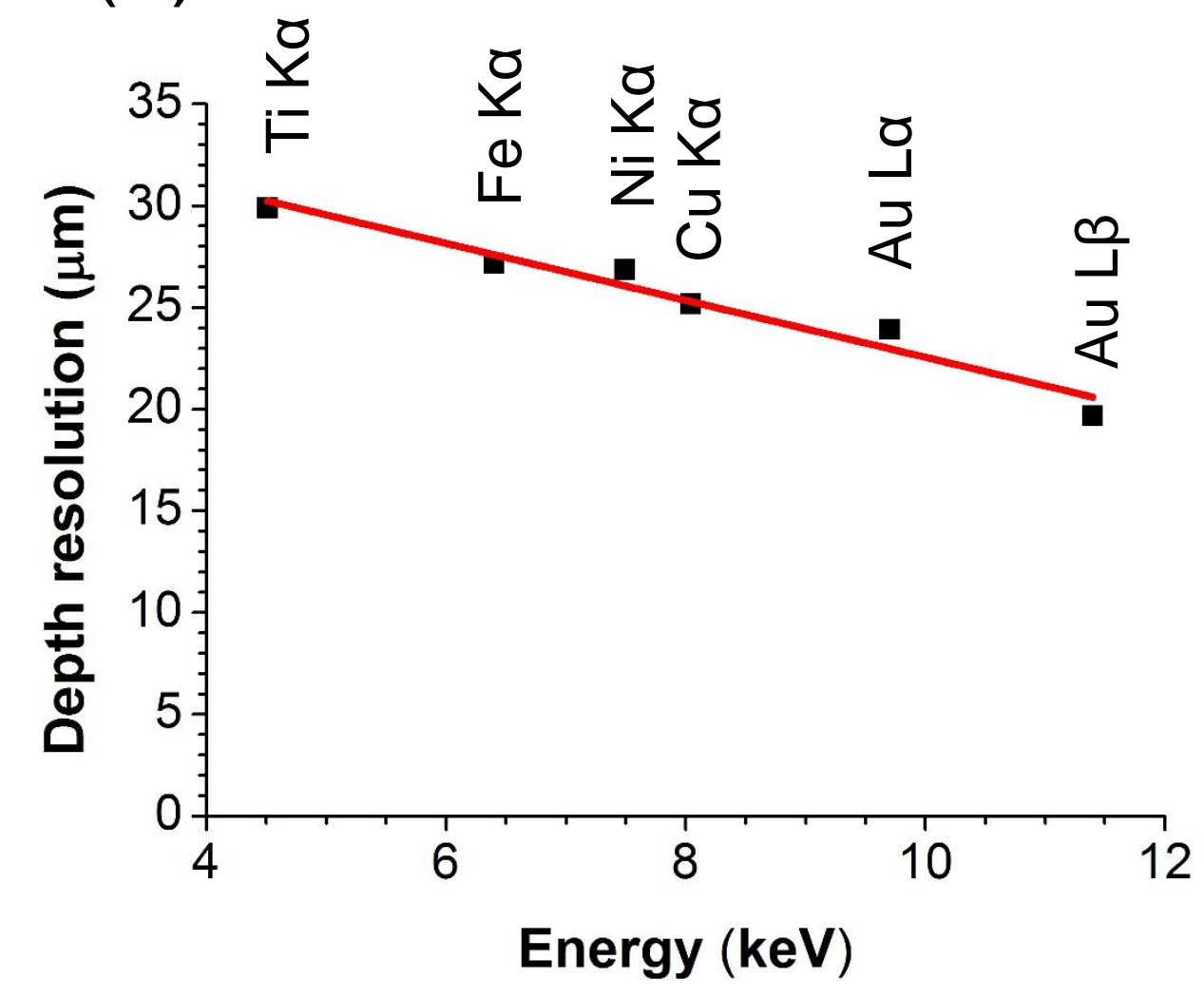

(b)

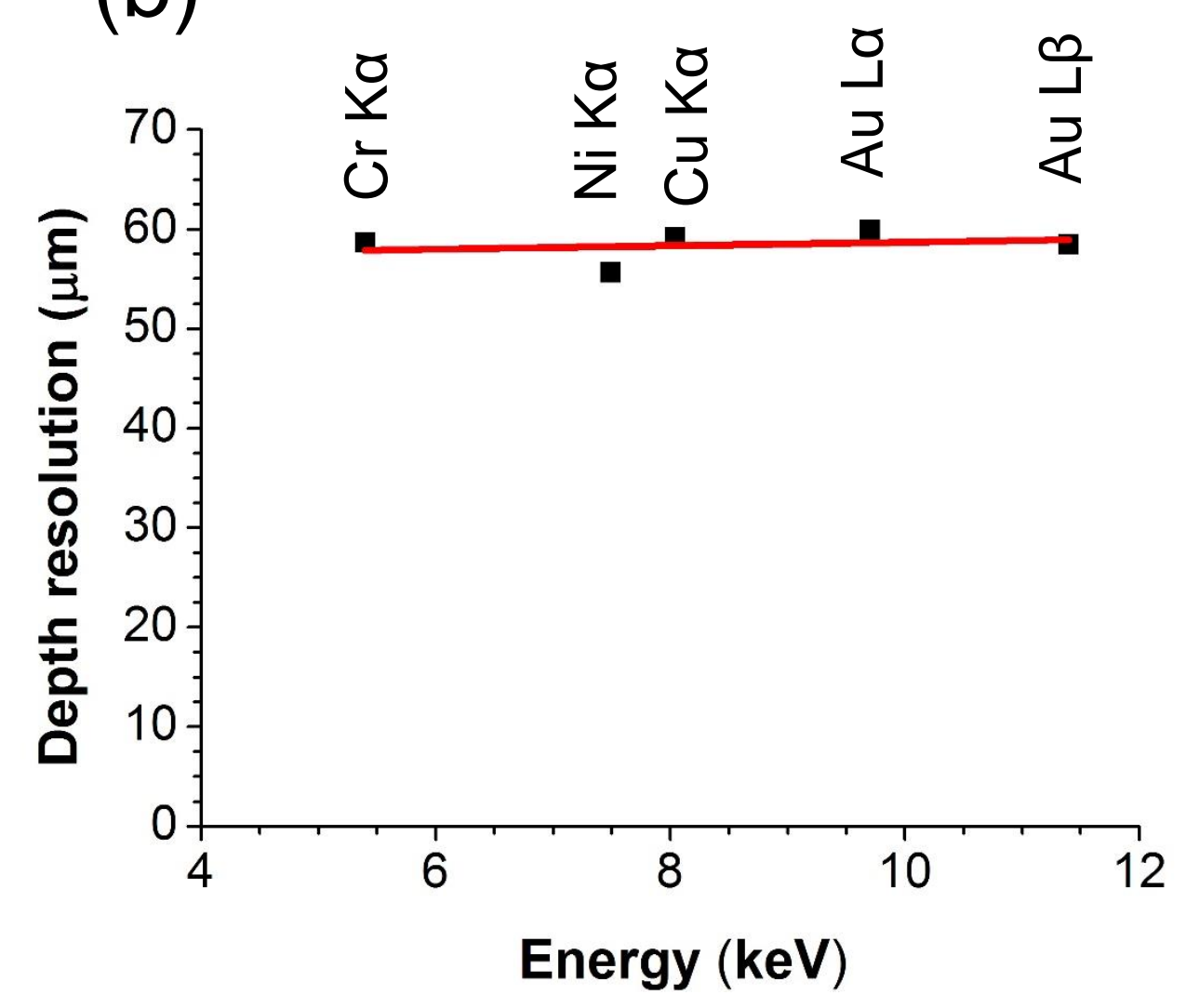

Fig. 8 Relationship between the X-ray energy and depth resolution using (a) C-P-XRF and (b) C-L-XRF. 
(a)
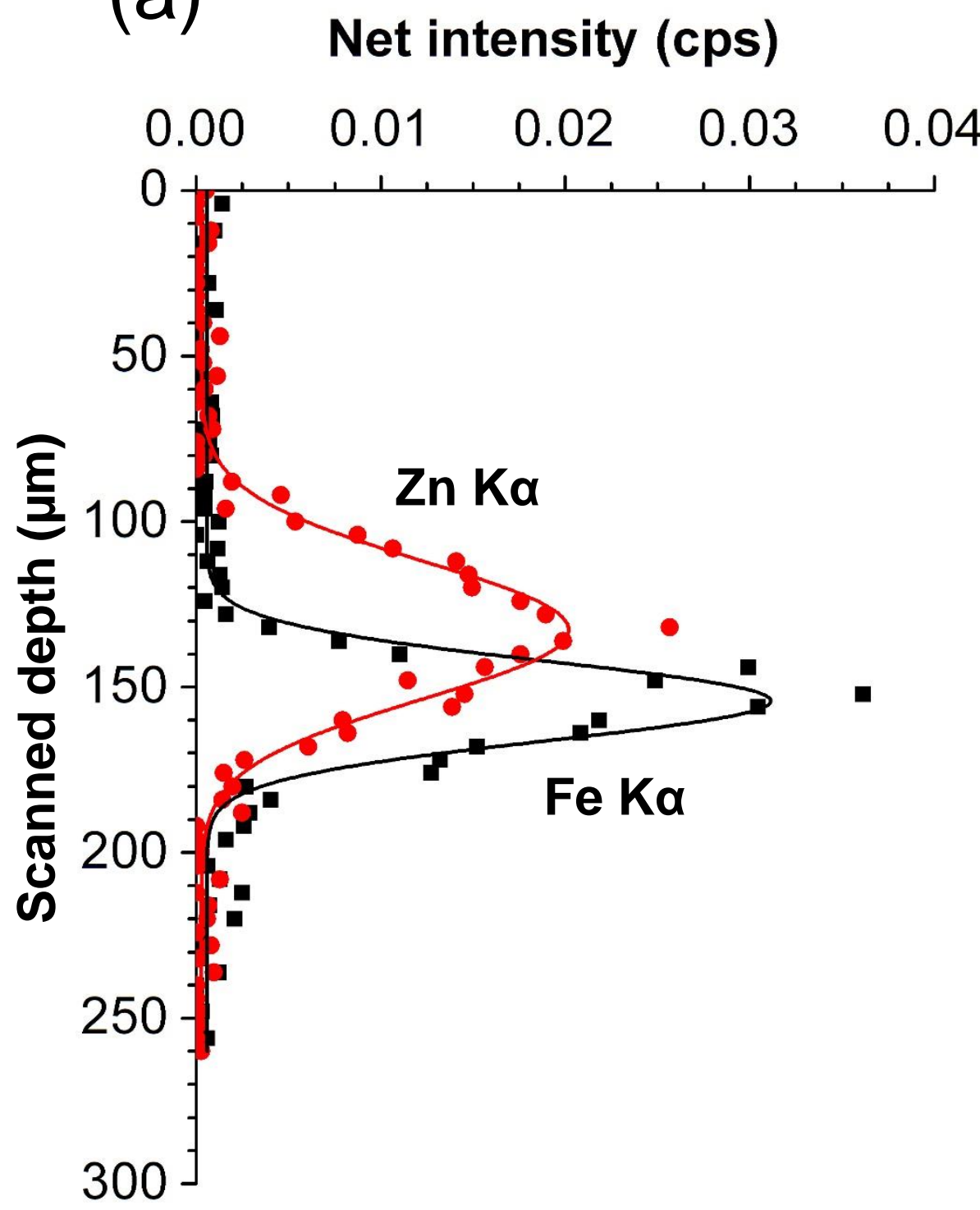

(b)

Net intensity (cps)

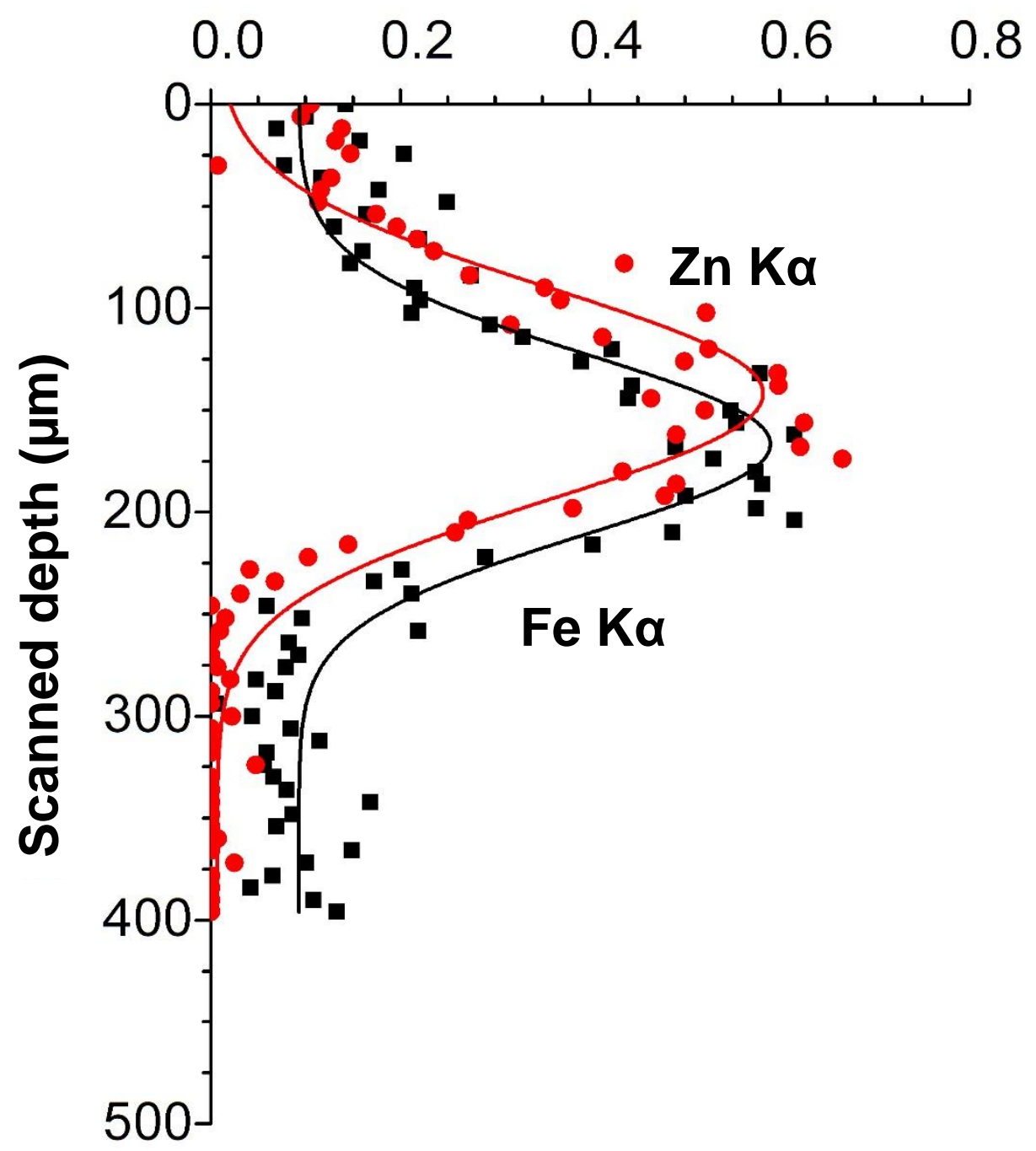

Fig. 9 Intensity profiles of the Fe K $\alpha$ and $\mathrm{Zn} \mathrm{K \alpha}$ intensities obtained using (a) C-P-XRF and (b) C-LXRF for the leather sample (Cordovan). Red symbols shows the $\mathrm{Zn} \mathrm{K \alpha}$ and black symbol shows the Fe $\mathrm{K} \alpha$ intensities. 